\title{
THE SCHUR MULTIPLIER OF A NILPOTENT GROUP
}

BY

\author{
URSULA MARTIN WEBB
}

\begin{abstract}
In this paper we obtain upper and lower bounds for the rank of the Schur multiplier of a nilpotent group in terms of the nilpotency class and the number of generators and rank of the derived quotient.
\end{abstract}

1. Introduction. The purpose of this paper is to discuss the torsion-free rank of the Schur multiplier $H_{2}(G, \mathbf{Z})=M(G)$ of the nilpotent group $G$. If $A$ is an abelian group, then $h(A)$ will denote the torsion-free rank of $A, h_{p}(A)$ the $p$-rank of $A$ where $p$ is a prime, and $d(A)$ the Prüfer rank of $A$, that is

$$
d(A)=h(A)+\max _{p} h_{p}(A) .
$$

If $G$ is a nilpotent group, then $h(G)$ will denote the torsion-free rank of $G$, that is the sum of the torsion-free ranks of each term of the lower central series of $G$. If $G$ is finitely generated so that $G_{\mathrm{ab}}=G /[G, G]$ is the product of a free abelian group $L$ and a finite group, then $d\left(G_{\mathrm{ab}}\right)$ is the minimum number of elements needed to generate $G_{\mathrm{ab}}$ or $G, h\left(G_{\mathrm{ab}}\right)$ is the rank of $L$, and $h\left(G_{\mathrm{ab}}\right)$ and $h(G)$ are finite.

We first consider upper bounds for $h(M(G))$.

THEOREM 1. Let $G$ be nilpotent of class $n$, and let $h=h\left(G_{\mathrm{ab}}\right)$.

(i) If $h(G)$ is finite, then $W_{h}(n+1) \geq h(M(G))$ and this bound is achieved by the free nilpotent group on $h$ generators of class $n$.

(ii) If $h(G)$ is infinite, then $h(G)=h\left(G_{\mathrm{ab}}\right)=h(M(G))$.

The value of $W_{h}(n+1)$ is given by Witt's formula

$$
W_{a}(b)=\left\{\sum_{k \mid b} a^{k} \mu\left(\frac{b}{k}\right)\right\} \cdot \frac{1}{b},
$$

where $\mu$ is the Möbius function.

Other upper bounds for $h(M(G))$ have been given in terms of $h(G)$. Stammbach $[10, \mathrm{p} .140]$ showed that if $h(G)$ is finite and $d=d\left(G_{\mathrm{ab}}\right)$ is finite, then

$$
h(M(G)) \leq(d-1) h(G)-\left(\begin{array}{l}
d \\
2
\end{array}\right)
$$

and Bacurin [2] showed that if $h(G)$ is finite, then

$$
h(M(G)) \leq\left(\begin{array}{c}
h(G) \\
2
\end{array}\right)
$$

Received by the editors November 18, 1983.

1980 Mathematics Subject Classification. Primary $20 \mathrm{~J} 05$. 
These bounds are both attained when $G$ is free abelian, when $h(G)=d$, and $h(M(G))=\left(\begin{array}{l}d \\ 2\end{array}\right)$.

Analogous upper bounds hold for finite $p$-groups; it was shown by Blackburn and Evens [3] that if $G$ is a finite $p$-group of class $c$ with $d\left(G_{\mathrm{ab}}\right)=d$, then $M(G)$ (which is a finite $p$-group) has $p$-rank at most $\sum_{j=1}^{c} W_{d}(j+1)$ and this bound is achieved by the free group on $d$ generators in a certain variety.

Turning to lower bounds we obtain the following result.

THEOREM 2. Let $G$ be nilpotent and suppose that $h(G)$ is finite. Let $h=$ $h\left(G_{\mathrm{ab}}\right)$. Then $h(M(G)) \geq f(h)$, where $f(h)$ denotes the minimum number of generators of $M(H)$ as $H$ ranges over all $h$ generator $p$-groups of exponent $p$ and class at most $p-1$, and $p$ ranges over all primes.

By estimating $f(h)$ using the Golod-Shafarevic inequality for finite $p$-groups we obtain

COROLlaRY 3. Let $G$ be nilpotent and suppose that $h(G)$ is finite. Let $h=$ $h\left(G_{\mathrm{ab}}\right)$. Then

$$
h(M(G))>\max \left(\frac{1}{4} h^{2}-h, h-2\right) .
$$

Notice that it follows from this that if $h\left(G_{\mathrm{ab}}\right)$ is at least two, then $M(G)$ is not trivial.

When $G$ has nilpotency class two, an alternative technique gives us a stronger bound.

THEOREM 4. Let $G$ be nilpotent of class two, and suppose that $h(G)$ is finite. Let $h=h\left(G_{\mathrm{ab}}\right)$. Then

$$
h(M(G)) \geq\left(h^{2}-1\right) / 3 .
$$

It seems unlikely that the lower bound in Corollary 3 is best possible, but by adapting an example due to Kostrikin [7] we see that the bound of Theorem 4 is sometimes attained.

THEOREM 5. Let $h$ be a power of two. Then there is a torsion-free nilpotent group $G$ of class two with $h\left(G_{\mathrm{ab}}\right)=h$ and $h(M(G))=\left(h^{2}-1\right) / 3$, which has a presentation on $h$ generators with $\left(h^{2}-1\right) / 3$ relations, such that any other presentation of $G$ needs $\left(h^{2}-1\right) / 3$ relations or more.

The lower bound for the $p$-rank of the multiplier of a finite $p$-group has been extensively discussed, partly because of its connection with the class field tower problem in number theory. It follows from the famous Golod-Shafarevic inequality (see Gruenberg [5] for example) that if $G$ is a finite $p$-group with $d\left(G_{\mathrm{ab}}\right)=d$, then

$$
d(M(G))>\frac{1}{4} d^{2}-d .
$$

This result has been strengthened by Gaschütz and Newman [4] who show in particular that if $G$ has class 2 , then

$$
d(M(G)) \geq d^{2} / 3+d / 6 .
$$

Although it is not known in general if either bound is best possible, the case of finite $p$-groups with trivial multiplier is of particular interest. Such groups must be cyclic or have $d=2$ or 3 , and examples are described in Wiegold [11] and Andozhskii [1]. 
When $G$ is finitely generated, $d(M(G))$ is closely related to $r(G)$, which is defined to be the minimum number of relators needed in any presentation of $G$. We shall show in $\S 2$ that

$$
r(G) \geq d\left(G_{\mathrm{ab}}\right)-h\left(G_{\mathrm{ab}}\right)+d(M(G))
$$

which together with the results above yields the following corollary. (Equation (1) is a variation of IV.6.1 of [10], which goes back to P. Hall.)

COROLLARY 6. Let $G$ be a finitely generated nilpotent group and let $h=$ $h\left(G_{\mathrm{ab}}\right)$. Then

(i) $r(G) \geq h(M(G))>\max \left(\frac{1}{4} h^{2}-h, h-2\right)$.

(ii) If $G$ has class two, then

$$
r(G) \geq h(M(G)) \geq\left(h^{2}-1\right) / 3
$$

and if $h$ is a power of two there is a group $G$ for which all three terms are equal.

The bound of (i) is somewhat weaker than that obtained by Lubotzky [8], who shows that if $G$ is any polycyclic-by-finite group with a presentation on generators $X$ with relators $R$ and $d\left(G_{\mathrm{ab}}\right)=d$, then

$$
|R| \geq|X|-d+d^{2} / 4-1 / 4 \text {. }
$$

If $G$ is soluble this gives $r(G) \geq d^{2} / 4-1 / 4$.

It is perhaps worth pointing out that no lower bound of Golod-Shafarevic type can exist for infinite nilpotent $p$-groups, as the following construction due to $\mathrm{D}$. Robinson shows. Let $Q$ be a finite $p$-group and let $\mu$ be an embedding of $M(Q)$ into $D$, a divisible abelian $p$-group. We regard $D$ as a trivial $Q$ module, and construct a central extension $G$ of $Q$ by $D$, with $M(G)=0$. Since $d\left(G_{\mathrm{ab}}\right)=r_{p}\left(G_{\mathrm{ab}}\right)$ can be arbitrarily large, we cannot hope to find a nonzero lower bound for $d(M(G))$. To construct $G$, observe that since $D$ is divisible, $\operatorname{Ext}\left(Q_{\mathrm{ab}}, D\right)=0$ and so the universal coefficients theorem gives

$$
H^{2}(Q, D) \cong \operatorname{Hom}(M(Q), D) .
$$

Thus there is a unique central extension $G$ of $D$ by $Q$ with cohomology class corresponding to $\mu$. Applying the six term homology sequence gives

$$
D \otimes G_{\mathrm{ab}} \rightarrow M(G) \rightarrow M(Q) \stackrel{\mu}{\rightarrow} D \rightarrow G_{\mathrm{ab}} \rightarrow Q_{\mathrm{ab}} \rightarrow 1
$$

and since $D \otimes G_{\mathrm{ab}}=0$ and $\mu$ is an embedding, $M(G)=0$.

The rest of the paper contains the following material. In $\S 2$ we introduce certain properties of the multiplier and prove a reduction lemma which reduces Theorems 1 and 2 to the finitely generated case. In $\S 3$ we prove Theorem 1 , in $\S 4$, Theorem 2 and Corollary 3 , and in $\S 5$ we consider groups of class two.

I should like to thank the referee for his careful reading of the manuscript.

2. Reduction to the finitely generated case. We shall use the following description of the multiplier, which can be obtained directly (see $[3,1.1]$ ) or from the five term sequence in homology. Let $Y$ be a normal subgroup of the group $X$, and suppose that $U$ and $S$ are normal subgroups of the free group $F$, with $F / U \cong X$ and $U S / U \cong Y$. Then the following sequence of abelian groups is exact:

$$
\begin{aligned}
1 & \rightarrow U \cap[S, F] /[U, F] \cap[S, F] \rightarrow M(X) \\
& \rightarrow M(X / Y) \rightarrow Y \cap[X, X] /[X, Y] \rightarrow 1 .
\end{aligned}
$$


We point out two special cases. If $X=Y$ we obtain the Hopf formula

$$
M(X) \cong U \cap[F, F] /[F, U] \text {. }
$$

If $G$ has nilpotency class $n$ so that $G=\gamma_{1}(G), \gamma_{i+1}(G)=\left[\gamma_{i}(G), G\right]$, and $\gamma_{n}(G) \neq$ $1, \gamma_{n+1}(G)=1$, then setting $X=G, Y=\gamma_{n}(G)$, and $S=\gamma_{n}(F)$ we obtain

$$
1 \rightarrow \gamma_{n+1}(F) /[F, U] \cap \gamma_{n+1}(F) \rightarrow M(G) \rightarrow M\left(G / \gamma_{n}(G)\right) \rightarrow \gamma_{n}(G) \rightarrow 1 .
$$

(Notice that $U \cap[S, F]=[S, F]$ because $U \geq \gamma_{n+1}(F)$.)

We can use the Hopf formula to write down equation (1). Suppose $G$ is finitely generated and $G=F / R$ is a presentation of $G$, where $F$ is free on $\alpha+d\left(G_{\mathrm{ab}}\right)$ generators for some $\alpha \geq 0$, and this presentation achieves the minimum number of relators. Then $R / R \cap[F, F] \cong R[F, F] /[F, F]$, which is torsion free of rank $\alpha+d\left(G_{\mathrm{ab}}\right)-h\left(G_{\mathrm{ab}}\right)$ since $F / R[F, F]$ has torsion free rank $h\left(G_{\mathrm{ab}}\right)$. Then the sequence of abelian groups

$$
1 \rightarrow R \cap[F, F] /[F, R] \rightarrow R /[F, R] \rightarrow R / R \cap[F, F] \rightarrow 1
$$

splits, so

$$
d(R /[F, R])=d(M(G))+d\left(G_{\mathrm{ab}}\right)-h\left(G_{\mathrm{ab}}\right)+\alpha .
$$

Now $r(G)$ is the minimum number of generators needed to generate $R$ as a normal subgroup of $F$, so $r(G) \geq d(R /[F, R])$ and

$$
r(G) \geq d(M(G))+d\left(G_{\mathrm{ab}}\right)-h\left(G_{\mathrm{ab}}\right) .
$$

We first prove a reduction lemma. A subgroup $N$ of a group $G$ is said to have $G: N$ torsion if some power of each element of $G$ lies in $N$.

LEMMA 1 (D. ROBINSON). Let $G$ be a nilpotent group and let $N \leq G$ where $G: N$ is torsion. Then $h(M(G))=h(M(N))$.

Proof. Suppose first that $N$ is normal in $G$, and consider the LyndonHochschild-Serre Spectral Sequence for the extension $1 \rightarrow N \rightarrow G \rightarrow Q \rightarrow 1$. Since $Q$ is locally finite and homology commutes with direct limits, $H_{i}(Q, A)$ is torsion for $i>0$ and for any module $A$. Thus $E_{i j}^{2}=H_{i}\left(Q, H_{j}(N, \mathbf{Z})\right)$ is torsion for $i>0$. Now $H_{2}(G, \mathbf{Z}) / F_{0} H_{2}(G, \mathbf{Z})$ is an extension of $E_{11}^{\infty}$ by $E_{20}^{\infty}$, both of which are torsion, so

$$
h(M(G))=h\left(F_{0} H_{2}(G, \mathbf{Z})\right)=h\left(E_{02}^{\infty}\right) .
$$

By considering $d_{2}$ and $d_{3}$ we see that $h\left(E_{02}^{\infty}\right)=h\left(E_{02}^{2}\right)=h\left(M(N)_{Q}\right)$. Now $G$ acts nilpotently on $N$, and hence on $M(N)$. Since $Q=G / N$ is torsion it must act trivially on the quotient of $M(N)$ by its torsion subgroup. Therefore $h\left(M(N)_{Q}\right)=$ $h(M(N))$. If $N$ is not normal it is certainly subnormal, since $G$ is nilpotent, and the result follows trivially.

To reduce the proofs of our first two theorems to the finitely generated case we shall use the following result.

LEMMA 2 (D. ROBINSON). Let $G$ be a nilpotent group. Then $G$ has a subgroup $N$ with $N_{\mathrm{ab}}$ free abelian, $h\left(N_{\mathrm{ab}}\right)=h\left(G_{\mathrm{ab}}\right)$ and $h(M(N))=h(M(G))$. Thus if $h\left(G_{\mathrm{ab}}\right)$ is finite, $N$ is finitely generated.

Proof. Let $X$ be a set of elements of $G$ of cardinality $h\left(G_{\mathrm{ab}}\right)$ which is linearly independent modulo $[G, G]$. Let $N=\langle X\rangle$. Clearly $h\left(N_{\mathrm{ab}}\right)=h\left(G_{\mathrm{ab}}\right)$ and $N_{\mathrm{ab}}$ 
is free abelian. It remains to show that $G: N$ is torsion: then by Lemma 1 , $h(M(N))=h(M(G))$. We shall show that $N \gamma_{i} / N \gamma_{i+1}$ is torsion for each $i \geq 1$, where $\gamma_{i}=\gamma_{i}(G)$. Since some $\gamma_{i} \subseteq N$, the result follows. By our choice of $N$, $N \gamma_{1} / N \gamma_{2}=G / N \gamma_{2}$ is torsion. Suppose that $N \gamma_{i-1} / N \gamma_{i}$ is torsion for some $i \geq 2$. If $x \in G$ and $y \in \gamma_{i-1}$, then by our induction hypothesis there exist integers $m$ and $n$ with $x^{m}$ and $y^{n} \in N \gamma_{i}$. Taken modulo $N \gamma_{i+1},[x, y]^{m n}=\left[x^{m}, y^{n}\right]$, and as $x^{m}$ and $y^{n}$ lie in $N \gamma_{i},\left[x^{m}, y^{n}\right]$ lies in $N \gamma_{i+1}$, and $[x, y]$ has finite order modulo $N \gamma_{i+1}$. Since $N \gamma_{i} / N \gamma_{i+1}$ is abelian and generated by elements of this form, it must be a torsion group.

We can now deduce part (ii) of Theorem 1. Observe first that if $G$ is nilpotent and $h\left(G_{\mathrm{ab}}\right)$ is an infinite cardinal $c$, then $h\left(\gamma_{i}(G) / \gamma_{i+1}(G)\right) \leq c$ for each $i$, so $h(G)=c$. By Lemma 2 we may pick a subgroup $N$ of $G$ with $h(M(G))=h(M(N)), N_{\text {ab }}$ free abelian and $h\left(N_{\mathrm{ab}}\right)=c$. Let $L$ be a normal subgroup of $N$ with $N / L=\langle x\rangle$ infinite cyclic, and let $M=L /[L, L]$. From Theorem 4.2 of $[3]$ we obtain the exact sequence

$$
M(G) \rightarrow H_{1}(\langle x\rangle, M)=M^{\langle x\rangle} \rightarrow 1 .
$$

Now $M^{\langle x\rangle}$ and $M /[M, x]$ have the same torsion-free ranks, since the exact sequence

$$
1 \rightarrow M^{\langle x\rangle} \rightarrow M \rightarrow[M, x] \rightarrow 1
$$

yields the exact sequence in homology

$$
1 \rightarrow[M, x]^{\langle x\rangle} \rightarrow M^{\langle x\rangle} \rightarrow M /[M, x] \rightarrow[M, x] /[M, x, x] \rightarrow 1 .
$$

By induction on the nilpotency class of $G$ the ranks of the terms at the two ends are equal, so the ranks in the middle are equal.

Hence

$$
\begin{aligned}
h(M(G)) \geq h\left(M^{\langle x\rangle}\right) & =h(M /[M, x])=h(L /[N, N]) \\
& =c-1=c
\end{aligned}
$$

and $h(M(G)) \geq c$.

On the other hand since $N_{\mathrm{ab}}$ is torsion free of rank $c$, we may assume that $N \cong F / R$ where $F$ has rank $c$. If $N$ has nilpotency class $n$ so that $R \geq \gamma_{n+1}$, then $M(G) \cong R /[F, R]$ is isomorphic to a subquotient of $F / \gamma_{n+2}(F)$, so $h(M(G)) \leq c$.

3. Proof of Theorem 1. By Lemma 2 we may assume that $G$ is finitely generated and that $G_{\mathrm{ab}}$ is free abelian of rank $h\left(G_{\mathrm{ab}}\right)=h$. We suppose that $G=F / R$ is a minimal presentation of $G$, so that $F$ is free on $h$ generators and $R \leq F^{\prime}$. We denote the terms of the lower central series of $F$ by $F_{i}=\gamma_{i}(F)$, and of $G$ by $G_{i}=\gamma_{i}(G)$. It is well known (see for example 5.11 of $[\mathbf{9}]$ ) that $F_{i} / F_{i+1}$ is free abelian of rank $W_{h}(i)$. Since $G$ has class $n, F_{n+1} \leq R$ and $F_{n} \not \leq R$.

The free nilpotent group of class $n$ on $h$ generators is isomorphic to $F / F_{n+1}$, and applying the Hopf formula we see that its multiplier is $F_{n+1} / F_{n+2}$, which has torsion-free rank $W_{h}(n+1)$. To obtain the upper bound we apply (2) with $R=S$ and $F_{n+1} \cong U$, so $X / Y=G$ and $X \cong F / F_{n+1}$. We obtain

$$
1 \rightarrow F_{n+1} \cap[F, R] / F_{n+2} \rightarrow M\left(F / F_{n+1}\right) \rightarrow M(G) \rightarrow Y \cap X^{\prime}[X, Y] \rightarrow 1 .
$$

It is clear that the right-hand term is isomorphic to $R /[R, F] F_{n+1}$. Let $a_{R}$ and $b_{R}$ be the torsion free ranks of the first and last terms of the sequence respectively, so 
that summing ranks we obtain

$$
a_{R}+h(M(G))=W_{h}(n+1)+b_{R} .
$$

Then it suffices to show that $a_{R} \geq b_{R}$.

Suppose that $R \leq F_{t}$ and $R \not F_{t+1}$ for some $t \geq 1$. We proceed by induction on $n+1-t$. If $n+1=t$, then $R=F_{n+1}$ and $a_{R}=b_{R}=0$. If $n+1-t=1$, then $[F, R] \leq F_{t+1}=F_{n+1}$ and we need to show that

$$
a_{R}=h\left([F, R] / F_{n+2}\right) \geq b_{R}=h\left(R / F_{n+1}\right) .
$$

Since $R \subseteq F_{2}$, we can choose a generator $f$ of $F$ so that $f R$ has infinite order modulo $R$. Consider the homomorphism

$$
[f,]: R / F_{n+1} \rightarrow[R, F] / F_{n+2}
$$

induced by $h \rightarrow[f, h]$ for all $h \in R$. It follows from Theorem 5.10 of [9] that any element of the kernel of this homomorphism lies in $R \cap\left\langle f, F_{n+1}\right\rangle / F_{n+1}$, which is trivial as $f$ has infinite order modulo $R$. Thus $[f$,$] is an embedding, and (5) holds.$

Suppose $n+1-t>1$, so that $n+1 \geq t+2 \geq 3$. Let $T=R \cap F_{t+1}$. Then $F_{n+1} \leq T \leq F_{t+1}$, so that we may assume $a_{T} \geq b_{T}$. Now

$$
a_{T}=h\left(F_{n+1} \cap[T, F] / F_{n+2}\right) \quad \text { and } \quad b_{T}=h\left(T /[T, F] F_{n+1}\right) .
$$

Evidently $a_{R} \geq a_{T}$ and

$$
b_{R}=b_{T}+h(R / T)-h\left([R, F] F_{n+1} /[T, F] F_{n+1}\right)
$$

so it suffices to show that

$$
h(R / T) \leq h\left([R, F] F_{n+1} /[T, F] F_{n+1}\right) .
$$

Again consider the map

$$
[f,]: R / T \rightarrow[R, T] F_{n+1} /[T, F] F_{n+1}
$$

for $f$ as before. If $k T$ lies in the kernel, then by 5.10 of [9]

$$
[f, k] \in[T, F] F_{n+1} \subseteq F_{t+2} F_{n+1} \subseteq F_{t+2},
$$

so

$$
k \in\left\langle f, F_{t+1}\right\rangle \cap R \subseteq\left\langle f, F_{t+1}\right\rangle \cap F_{2} \subseteq F_{t+1} \cap R=T .
$$

Thus $[f$,$] is an embedding and (6) holds.$

4. Proof of Theorem 2. The proof of Theorem 2 will arise from considering a certain finite quotient of the group $G$. If $G$ is any group and $p$ is a prime, then $G^{p}$ denotes $\left\langle x^{p} \mid x \in G\right\rangle$. We shall need the following lemma.

LEMMA 3. Suppose that $G$ is nilpotent of class $n$ and that $p$ is a prime with $p>n$ such that $G_{\mathrm{ab}}$ has no elements of order $p$. Then

$$
G^{p} \cap[G, G]=\left[G^{p}, G\right] .
$$

ProOF. We use the Hall Petrescu formula [6, p. 317], which states that if $x$ and $y$ are elements of any group, then

$$
x^{p} y^{p}=(x y)^{p} c_{2}^{p} \cdots c_{p-1}^{p} c_{p},
$$


where $c_{i} \in \gamma_{i}\langle x, y\rangle$. Of course in $G, c_{p}=1$. Evidently $\left[G^{p}, G\right] \subseteq G^{p} \cap[G, G]$. We shall show that

$$
G^{p} \cap[G, G] \subseteq[G, G]^{p} \subseteq\left[G^{p}, G\right] .
$$

So suppose that $u \in G^{p} \cap[G, G]$. Then $u$ is a product of $p$ th powers, and by (7) $u$ is a $p$ th power modulo $[G, G]^{p}$. As $G_{\text {ab }}$ has no elements of order $p, u$ must be a $p$ th power of an element of $[G, G]$ modulo $[G, G]^{p}$. Hence $G^{p} \cap[G, G] \subseteq[G, G]^{p}$.

Now we show that $\gamma_{i}^{p} \subseteq\left[G^{p}, G\right]$ for each $i$, where $\gamma_{i}=\gamma_{i}(G)$. Clearly $1=$ $\gamma_{p}^{p} \subseteq\left[G^{p}, G\right]$. So suppose that $\gamma_{i+1}^{p} \subseteq\left[G^{p}, G\right]$ for some $i \geq 2$. It follows from (7) that $\gamma_{i}^{p}$ is generated moduo $\gamma_{i+1}^{p}$ by elements of the form $[x, y]^{p}$ where $x \in \gamma_{i-1}$, $y \in G$. We have $[x, y]^{p}=\left(x^{-1} x^{y}\right)^{p}=x^{-p}\left(x^{p}\right)^{y} c_{2}^{p} s$, where $s \in \gamma_{i+1}^{p} \subseteq\left[G^{p}, G\right]$ and $c_{2} \in \gamma_{2}\left\langle x^{-1}, x^{y}\right\rangle$. Now

$$
\left[x^{-1}, x^{y}\right]=\left[x^{-1}, x[x, y]\right]=\left[x^{-1},[x, y]\right] \in \gamma_{f},
$$

where $f=2(i-1)+1 \geq i+1$ for $i \geq 2$, so $c_{2}^{p} \in \gamma_{f}^{p} \subseteq\left[G^{p}, G\right]$. Thus $[x, y]^{p} \in\left[G^{p}, G\right]$, so $\gamma_{i}^{p} \subseteq\left[G^{p}, G\right]$ and the result follows.

Now we can prove Theorem 2.

ProOF OF THEOREM 2. By Lemma 2 we may assume that $G$ is finitely generated and $G_{\mathrm{ab}}$ is free abelian of rank $h$. Let $p$ be a prime greater than $n$, the class of $G$, such that $M(G)$ has no elements of order $p$. (Of course there are infinitely many choices for $p$.) We apply sequence (2) of $\S 2$ with $X=G, Y=G^{p}$ to obtain

$$
M(G) \stackrel{\alpha}{\rightarrow} M\left(G / G^{p}\right) \rightarrow G^{p} \cap G^{\prime} /\left[G^{p}, G\right] \rightarrow 1 .
$$

By Lemma 3 and our choice of $p, G^{p} \cap G^{\prime} /\left[G^{p}, G\right]=1$, so $\alpha$ is surjective. Now $M\left(G / G^{p}\right)$ is a $p$-group and $M(G)$ has no elements of order $p$, so that $d\left(M\left(G / G^{p}\right)\right) \leq$ $h(M(G))$. Since $d\left(G / G^{\prime} G^{p}\right)=h$ and $G / G^{p}$ has class at most $p-1$, it follows that $h(M(G)) \geq f(h)$.

PROOF OF COROLlaRY 3. To deduce this we consider the function $f(h)$. The Golod-Shafarevic inequality gives $f(h)>h^{2} / 4-h$. The other part of the bound is a consequence of the following lemma.

LEMMA 4. Let $H$ be a group of exponent $p$ and class at most $p-1$ on $h$ generators. Then $d(M(G)) \geq h-1$.

Proof. Let $H=\langle L, x\rangle$, where $|G: L|=p$. Then $H$ splits over $L$ and we have (either from the spectral sequence or $[3,4.2]$ )

$$
M(G) \rightarrow H_{1}\left(\langle x\rangle, L_{\mathrm{ab}}\right) \rightarrow 0 .
$$

Of course $L_{\mathrm{ab}}$ has exponent $p$, and as $\langle x\rangle$ module is the direct sum of $h-1$ indecomposable summands. Recall that $\langle x\rangle$ has $p$ isomorphism types of indecomposable modules over $G F(p), V_{1}, \ldots, V_{p}$ where $\left|V_{i}\right|=p^{i}$, and that $\left|H_{1}\left(\langle x\rangle, V_{i}\right)\right|=p$ for $i<p$. Since $G$ has class at most $p-1, V_{p}$ cannot occur as a summand of $L_{\mathrm{ab}}$, and since homology commutes with direct sums, $d\left(H_{1}\left(\langle x\rangle, L_{\mathrm{ab}}\right)\right)=h-1$. Thus $d(M(G)) \geq h-1$.

5. The class two case. The proof of Theorem 4 uses a different technique, which seems difficult to generalise to higher nilpotency class.

PROOF OF THEOREM 4. By Lemma 2 we may assume that $G_{\mathrm{ab}}$ is free abelian, so that if $G=F / R$ is a minimal presentation of $G$, where $F$ is free of rank $h$, then 
$R \subseteq \gamma_{2}(F)$. Let $\gamma_{i}=\gamma_{i}(F)$. Since $G$ has class two, $\gamma_{3} \subseteq R$ and so $\gamma_{4} \subseteq[F, R] \subseteq \gamma_{3}$. Now $[F, R] / \gamma_{4}$ is a homomorphic image of $F_{\mathrm{ab}} \otimes R / \gamma_{3}$, so $h\left([F, R] / \gamma_{4}\right) \leq h \cdot h\left(R / \gamma_{3}\right)$. We have from the Hopf formula

$$
\begin{aligned}
h(M(G)) & =h(R /[F, R])=h\left(R / \gamma_{3}\right)+h\left(\gamma_{3} /[F, R]\right) \\
& \geq\left(h\left(\gamma_{3} / \gamma_{4}\right)-h\left(\gamma_{3} /[F, R]\right)\right) / h+h\left(\gamma_{3} /[F, R]\right) \\
& \geq 1 / h \cdot W_{h}(3)=\left(h^{2}-1\right) / 3 .
\end{aligned}
$$

Finally we prove Theorem 5 .

PROOF OF THEOREM 5. We shall exhibit a group on $h=2^{n}$ generators with $\left(h^{2}-1\right) / 3$ relators, and show that it is torsion free nilpotent of class two. Then we have using (1)

so

$$
\frac{h^{2}-1}{3} \geq r(G) \geq h(M(G)) \geq \frac{h^{2}-1}{3}
$$

$$
r(G)=h(M(G))=\frac{h^{2}-1}{3} .
$$

Let $B_{t}$ denote all binary representations of length $t$, so that a typical element of $B_{t}$ is a string $\alpha_{1} \cdots \alpha_{t}$ of zeros and ones. We suppose that $G$ is generated by $\left\{g_{\alpha} \mid \alpha \in B_{n}\right\}=X$. For $1 \leq k \leq n$ and $\alpha, \beta \in B_{n-k}$ define

$$
W(\alpha, \beta)=\prod_{\gamma}\left[g_{\gamma 0 \alpha}, g_{\gamma 1 \beta}\right]
$$

where the product is taken over all $\gamma$ in $B_{k-1}$, in any order.

Let $S_{k}=\left\{W(\alpha, \beta) \mid \alpha, \beta \in B_{n-k}\right\}$, let $S=\bigcup_{k=1}^{n} S_{k}$ and let $G=\langle X \mid S\rangle$. Notice that $\left|S_{k}\right|=4^{n-k}$ and $|S|=1+\cdots+4^{n-1}=\left(d^{2}-1\right) / 3$. In particular $S_{1}$ consists of all commutators of the form

$$
\left[g_{0 \alpha}, g_{1 \beta}\right]
$$

with $\alpha, \beta \in B_{n-1}$, and $S_{n}$ has just one element

$$
\prod_{\gamma \in B_{n-1}}\left[g_{\gamma 0}, g_{\gamma 1}\right]
$$

To show that $G$ is nilpotent of class two we need to show that all commutators of the form $\left[\left[g_{\alpha}, g_{\beta}\right] g_{\gamma}\right]$ with $\alpha, \beta, \gamma \in B_{n}$ are trivial. We shall use the Witt identity in the form

$$
[[a, b] c][[b, c] a][[c, a] b]=[b, a][c, a][c, b]^{a}[a, b][a, c]^{b}[b, c]^{a}[a, c][c, a]^{b} .
$$

Recall also that $[x, y]^{-1}=[y, x]$, and that

$$
[a b, c]=[a, c]^{*}[b, c]
$$

where $[a, c]^{*}$ denotes some conjugate of $[a, c]$.

So suppose that $\left[\left[g_{\alpha}, g_{\beta}\right] g_{\gamma}\right] \neq 1$ and $\alpha=\alpha_{1} \cdots \alpha_{n}, \beta=\beta_{1} \cdots \beta_{n}$ and $\gamma=$ $\gamma_{1} \cdots \gamma_{n}$. We may assume from (8) that $\alpha_{1}=\beta_{1}$, as otherwise $\left[g_{\alpha}, g_{\beta}\right]=1$. Suppose $\gamma_{1} \neq \alpha_{1}$. By (8) $g_{\gamma}$ commutes with $g_{\alpha}$ and $g_{\beta}$, so hence with $\left[g_{\alpha}, g_{\beta}\right]$, which is false. So $\alpha_{1}=\beta_{1}=\gamma_{1}$.

Now suppose that we have shown $\left[\left[g_{\alpha}, g_{\beta}\right] g_{\gamma}\right]=1$ unless $\alpha_{1} \cdots \alpha_{k-1}=\beta_{1} \cdots \beta_{k-1}$ $=\gamma_{1} \cdots \gamma_{k-1}$ for some $2 \leq k \leq n$. 
Case 1. Suppose $\alpha_{k} \neq \beta_{k}$. Then $\left[g_{\alpha}, g_{\beta}\right]$ occurs in $w$, a relator in $S_{k}$ or the inverse of a relator in $S_{k}$, of which the other terms have the form $\left[g_{\rho}, g_{\sigma}\right]$ with $\rho_{1} \rho_{2} \cdots \rho_{k-1}=\sigma_{1} \cdots \sigma_{k-1} \neq \alpha_{1} \cdots \alpha_{k-1}$, so by our induction hypothesis each such $\left[\left[g_{\rho}, g_{\sigma}\right] g_{\gamma}\right]=1$. After repeated application of $(10)$, we obtain

$$
\begin{aligned}
1=\left[w, g_{\gamma}\right] & =\prod_{\rho, \sigma}\left[\left[g_{\rho}, g_{\sigma}\right] g_{\gamma}\right]^{*} \cdot\left[\left[g_{\alpha}, g_{\beta}\right] g_{\gamma}\right]^{*} \cdot \prod_{\rho, \sigma}\left[\left[g_{\rho}, g_{\sigma}\right] g_{\gamma}\right]^{*} \\
& =\left[\left[g_{\alpha}, g_{\beta}\right] g_{\gamma}\right] .
\end{aligned}
$$

Case 2. Suppose $\alpha_{k}=\beta_{k} \neq \gamma_{k}$. By Case 1 ,

$$
1=\left[\left[g_{\alpha}, g_{\gamma}\right] g_{\alpha}\right]=\left[\left[g_{\alpha}, g_{\gamma}\right] g_{\beta}\right]=\left[\left[g_{\beta}, g_{\gamma}\right] g_{\alpha}\right]=\left[\left[g_{\beta}, g_{\gamma}\right] g_{\beta}\right]
$$

and so

$$
\left[\left[g_{\gamma}, g_{\alpha}\right],\left[g_{\alpha}, g_{\beta}\right]\right]=\left[\left[g_{\gamma}, g_{\beta}\right],\left[g_{\alpha}, g_{\beta}\right]\right]=\left[\left[g_{\gamma}, g_{\beta}\right],\left[g_{\alpha}, g_{\gamma}\right]\right]
$$

and applying (9) with $a=g_{\alpha}, b=g_{\beta}, c=g_{\gamma}$ we obtain $\left[\left[g_{\alpha}, g_{\beta}\right] g_{\gamma}\right]=1$.

Thus we have $\alpha_{k}=\beta_{k}=\gamma_{k}$. Continuing we get $\alpha=\beta=\gamma$, when of course $\left[\left[g_{\alpha}, g_{\beta}\right] g_{\gamma}\right]=1$ and the result follows.

NOTE. Kostrikin in [7] uses a Lie ring argument similar to this to show that the group above with the additional relations $g_{\alpha}^{p}=1$ for each $\alpha \in B_{n}$, where $p$ is an odd prime, has exponent $p$, class two and $h+\left(h^{2}-1\right) / 3$ generators.

\section{REFERENCES}

1. A. V. Andozhskii, On some classes of closed pro-p groups, Izv. Akad. Nauk SSSR Ser. Mat. 39 (1975), 707-738.

2. Y. Bacurin, The multipliers of torsion-free nilpotent groups, Mat. Zametki 5 (1969), 541-544.

3. N. Blackburn and L. Evens, Schur multipliers of p-groups, J. Reine Angew. Math. 309 (1979), 100-113.

4. W. Gaschütz and M. Newman, On presentations of finite p-groups, J. Reine Angew. Math. 245 (1970), 172-176.

5. K. W. Gruenberg, Cohomological topics in group theory, Lecture Notes in Math., vol. 143, Springer, Berlin, 1970.

6. B. Huppert, Endliche Gruppen, Springer, Berlin, 1967.

7. A. I. Kostrikin, On presenting groups by generators and defining relations, Izv. Akad. Nauk SSSR Ser. Mat. 29 (1965), 1119-1122.

8. A. Lubotzky, Group presentation, p-adic analytic groups and lattices in $\mathrm{SL}_{2}(\mathrm{C})$ (to appear).

9. W. Magnus, A. Karrass and D. Solitar, Combinatorial group theory, Dover, New York, 1976.

10. U. Stammbach, Homology in group theory, Lecture Notes in Math., vol. 359, Springer, Berlin, 1973.

11. J. Wiegold, The Schur multiplier. an elementary approach, Groups-St. Andrews 1981 (Eds., C. M. Campbell and E. Robertson), Cambridge Univ. Press, Cambridge, 1982.

Department of Computation, UMiSt, Manchester M60 1QD, England 